\title{
IMPROVED TECHNIQUES FOR POWER SYSTEM VOLTAGE STABILITY ASSESSMENT USING ENERGY METHODS
}

\author{
Thomas J. Overbye \\ Student Member, IEEE \\ Christopher L. DeMarco \\ Member, IEEE \\ Department of Electrical and Computer Engineering \\ University of Wisconsin-Madison \\ Madison, WI 53706
}

\begin{abstract}
This paper presents an improved method of assessing power system voltage stability using energy techniques. The concept of an energy function providing a localized measure of voltage security in a particular portion of the system is developed. Also an improved method of locating power flow alternative solutions with low associated energy measures is presented. Techniques are demonstrated on IEEE 118 bus system and a 415 bus system.
\end{abstract}

KEYWORDS: Voltage stability, voltage collapse, power flow.

\section{Introduction}

Increased loads and larger inter-utility power transfers coupled with fewer additions to the transmission system have resulted in an increasing danger of voltage instability and collapse for many utilities. Actual incidents of voltage collapse indicate the potential magnitude of the problem [1],[2]. Also, an imprecisely quantified threat of voltage collapse often constrains economic operations of systems to maintain reliability [3],[4]. In order to operate systems with maximum economy and reliability, utilities need an accurate indicator of the proximity of their system to voltage collapse.

A large portion of recent literature analyzing the problems of voltage instability and voltage collapse has focused on the use of multiple power flow solutions, and in more formal mathematical analyses, on bifurcation phenomena in which multiple solutions approach one another and ultimately disappear under the influence of changing network parameters. The non-uniqueness of power flow solutions is first discussed in depth in [5]. Usually only one of the power flow solutions corresponds to a practical stable equilibrium point (SEP) for the power system. This solution will be denoted as the "operable" solution. The other solutions typically correspond to unstable equilibrium points (UEPs) of the power system. For voltage collapse analysis, these solutions are often characterized by low bus voltage magnitudes, and will be referred to as the "low voltage" solutions. An algorithm for locating multiple solutions is presented in [6]. The relationship between voltage instability and multiple solutions is shown in [7]. In [8] and later in [9] concepts of static bifurcation theory are used to formally show the relationship between bifurcation of the power flow equations in a multibus system and voltage instability

91 WM 094-3 PWRS A paper recommended and approved by the IEEE Power System Engineering Committee of the IEEE Power Engineering Society for presentation at the IEEE/PES 1991 Winter Meeting, New York, New York, February 3-7, 1991. Manuscript submitted August 24, 1990; made available for printing January 22, 1991.
More recently in [10] and [11] indices which attempt to quantify the distance between the operable solution and a low voltage solution are presented. In [12] a technique for rapidly locating a pair of closely located power flow solutions is introduced.

The use of energy function methods to determine proximity to voltage collapse is presented by the authors in [13] and [14]. In this method, a closed form voltage security measure (or energy measure) is defined by integrating a power mismatch expression between the operable solution and a low voltage solution. A crucial factor in the use of the energy function method is the ability to rapidly determine the appropriate low voltage solution to use in the energy measure calculation.

The goal of this paper is to address the determination of these low voltage solutions, and consequently to demonstrate the practicality of using energy methods to assess voltage security in a large scale power system. The paper is organized as follows. First, the use of energy function methods in the problem of voltage collapse is discussed, with emphasis on the attributes of the low voltage solutions. Second, an algorithm to rapidly locate the appropriate low voltage solutions in large scale systems is presented. Lastly, calculation of these solutions and use of the energy method to predict proximity to collapse is demonstrated in the IEEE 118 bus system and in a 415 bus system.

\section{Energy Methods and the Role of Alternate Power} Flow Solutions in Voltage Collapse Studies

The system model and energy function expression to be used here are described in detail in [13] and [14]. As a review, the use of energy methods to determine system vulnerability to voltage collapse is briefly described below. For reference, the energy function expression from [14] is included in the Appendix. A scalar energy function dependent on system state (system bus voltage magnitudes and phase angles) is first defined, with the property that the operable solution defines a local minimum of this energy. Formally, the energy function is said to be positive definite about the operating point; intuitively, one may think of the operating point as occupying the bottom of an "energy well." A key step in such energy based stability studies is to identify those unstable equilibrium points (UEPs) that form saddlepoints on the boundary of the energy well. In the framework to be discussed here, the UEPs of interest will correspond to alternate power flow solutions that display low voltage magnitudes at certain buses. Conceptually the energy function shows the height of the "potential barrier" (or equivalently the depth of the energy well) between the operable solution and a low voltage solution.

To understand the use of such energy analysis in voltage stability studies, it is important to recognize that the instantaneous state of the system does not sit precisely at the stable equilibrium point predicted by the power flow. The system state is constantly being perturbed by small, random changes in time varying loads. These perturb the system state away from equilibrium, and can be viewed as introducing a small amount of energy into the system. 
These disturbances are normally dissipated through system damping, so that at a secure operating point, where the energy well is relatively deep, their effect is negligible. However, as the system evolves towards an operating point vulnerable to voltage collapse, the depth of the energy well decreases. Eventually a point is reached where the disturbance energy is sufficient for the state to escape this well, with a resultant voltage collapse. Work reported in [13] and [14] argued that the depth of the energy well (as measured by the energy difference between the operable solution and a low voltage power flow solution) can provide an excellent indicator of the system's vulnerability to voltage collapse.

It is interesting to note that the energy function defined here considers both voltage and angle deviations from equilibrium. However, the use of low voltage power flow solutions implies that the type of instabilities studied here will be characterized by motion of the state in a direction that causes significant drops in some bus voltage magnitudes, with relatively small changes in phase angles. If other types of UEPs were examined (for example, those UEPs which yield large angular differences across cutsets of transmission lines), the energy might be interpreted as a security measure for certain types of angular instabilities. However, these types of angular instabilities and their associated UEPs will not be examined here.

The energy difference is clearly dependent on the low voltage solution selected. For large systems the choice of which low voltage solution to use can be difficult. In a lightly loaded, $n$ bus system there may exist up to $2^{n-1}$ solutions of the power flow equations [15],[16]. While experience at realistic loading levels has shown that the number is often substantially less, it is still imperative that a numerically efficient method be found for determining the appropriate set of low voltage solutions to use for assessing system voltage security.

As the system parameters (typically loads and generation) move quasi-statically toward the point of voltage collapse, the number of low voltage solutions tends to decrease [17]. At the point immediately before collapse only the operable solution and a single low voltage solution exist. These two solutions eventually coalesce, with resultant loss of the steady state equilibrium point. In [18] it is shown that for typical power system models, the system always loses steady state stability by a saddle-node bifurcation between the operable solution and a type-one low voltage solution. A type-one solution has the property that the linearized system about that equilibrium point has a single positive eigenvalue. Therefore the set of low voltage solutions to be examined can be restricted to those which are type-one.

\section{Predicting Areas of Voltage Collapse through} Properties of Low Voltage Power Flow Solutions

Classifying solutions as type-one raises the computational issue of identifying the stability properties of a power flow solution with respect to the linearized dynamic model. A number of authors have examined the issue of relating small disturbance stability of the linearized power system dynamics to the eigenvalues of the power flow Jacobian (see [19] and references therein). For a lossless version of the model employed here, the system energy function is positive definite about an equilibrium when the power flow Jacobian has all negative eigenvalues. With additional results established in [20], this in turn implies that the dynamic model is stable. Hence, small disturbance stability of the dynamic model can be guaranteed by examining the eigenvalues of the power flow Jacobian. While this result formally holds true only in the lossless case, eigenvalues of both the power flow
Jacobian and those of the dynamic linearization vary continuously with respect to the conductance terms. With a simple eigenvalue perturbation analysis, one can show that if the lossless system stability prediction has a reasonable margin (i.e., the power flow Jacobian eigenvalues are all less than some negative threshold), then this stability prediction carries through to the more exact dynamic model with non-zero conductances. The reader should note that these results need to be qualified by the assumption that the exciter control loop is stable. As discussed in [19], if the flux decay dynamics and excitation control loop introduce instabilities, then the linearized dynamic models may be unstable even though the power flow Jacobian has all negative eigenvalues. The model employed here used a type of fast exciter representation with saturation (see [14] for details) that implicitly assumes the voltage control loop is stable. Hence, this type of exciter instability is not treated here.

At the point of bifurcation between the SEP solution (denoted by $x^{0}$ ) and a type-one UEP (denoted as $x^{1}$ ), one has $x^{0}=x^{1}=$ $\mathbf{x}^{*}$, and the SEP loses asymptotic stability with its Jacobian having a zero eigenvalue, $\lambda^{1}=0$. A slight further perturbation would then result in divergence of the system state away from the operating point. In [18] and [21] it is shown that the initial direction of the voltage collapse is along the eigenvector $v^{1}=v^{*}$ corresponding to the zero eigenvalue of the Jacobian at $x^{1}$. The magnitudes of the individual components of $v^{1}$ indicate how rapidly the voltage or angle at each individual bus will initially diverge relative to the other system buses. Generally the magnitude of the initial voltage drop or angle slip is significant only at a subset of the system buses. Therefore while voltage collapse is characterized by loss of a steady state equilibrium for the entire system, its initial effects are normally apparent only at a subset of the system buses. Since these buses are usually electrically close to each other, the subset will be referred to as an area. This set of affected buses will be denoted as Area $\left(\mathbf{x}^{1}\right)$. The extent to which a voltage collapse propagates depends upon the amount and location of the protective equipment on the system.

Now assume that the system has not yet reached the point where bifurcation is predicted mathematically (i.e., $x^{0}$ and $x^{1}$ are still separate). Given that the system varies quasi-statically, whether $x^{0}$ and $x^{1}$ will eventually coalesce depends on the future variation in system parameters (e.g. loads and generation). Since $\mathbf{x}^{1}$ is a type-one equilibrium, the eigenvector $\mathbf{v}^{1}$ associated with the positive eigenvalue of its Jacobian can be calculated. Interestingly, numerical tests in a number of test systems indicate that the relative sizes of the components of $\mathbf{v}^{1}$ are fairly insensitive to the distance between $x^{0}$ and $x^{1}$ (with the energy difference being used as a distance function). Even while the two solutions are quite far apart in state space, the components of $\mathbf{v}^{1}$ approximate how the system would collapse if the variation of the system parameters was such that $x^{0}$ and $x^{1}$ eventually coalesce. Thus by defining $\vartheta\left(\mathbf{x}^{1}\right)$ to be the energy difference between the low voltage solution $x^{1}$ and the operable solution $x^{0}, \vartheta\left(x^{1}\right)$ can be interpreted as a proximity indicator for voltage collapse in Area $\left(\mathbf{x}^{1}\right)$. When a system has more than a single type-one low voltage solution, a separate energy measure could be calculated for each Area $\left(\mathbf{x}^{\mathbf{i}}\right)$.

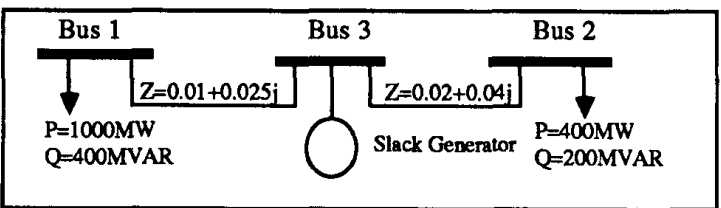

Figure 3-1 
To illustrate the role of the unstable eigenvector in predicting the area of collapse, consider the simple system of Figure 3-1. This system consists of a strong generator bus (an infinite or slack bus), with two separate load buses connected through transmission lines. Mathematically, the system is equivalent to two independent two bus systems. Since each of these independent two bus systems can have at most two solutions [6], it is clear that for the three bus system there are at most four power flow solutions. For the loads shown in Figure 3-1. Table 3-1 shows the four solutions, their associated energy, and where appropriate, the unstable eigenvector of the power flow Jacobian.

\begin{tabular}{|c|c|c|c|c|}
\hline & & jlution Numb & & \\
\hline & 0 & 1 & 2 & 3 \\
\hline Bus 1 Voltage & 0.614 & 0.472 & 0.614 & 0.472 \\
\hline Bus 1 Angle & $-20.0^{\circ}$ & $-26.4^{\circ}$ & $-20.0^{\circ}$ & $-26.4^{\circ}$ \\
\hline Bus 2 Voltage & 0.784 & 0.784 & 0.255 & 0.255 \\
\hline Bus 2 Angle & $-8.8^{\circ}$ & $-8.8^{\circ}$ & $-28.1^{\circ}$ & $-28.1^{\circ}$ \\
\hline Energy measure & & 0.127 & 1.636 & 1.763 \\
\hline $\begin{array}{l}\text { Jacobian } \\
\text { eigenvalues }\end{array}$ & $\begin{array}{c}-4.86 \\
-26.79 \\
-13.72+5.24 \mathbf{j} \\
-13.72-5.24 j\end{array}$ & $\begin{array}{c}4.22 \\
-23.74 \\
-13.72+5.24 j \\
-13.72-5.24 j\end{array}$ & $\begin{array}{l}8.09 \\
-8.65 \\
-4.86 \\
-26.79\end{array}$ & $\begin{array}{c}4.22 \\
8.09 \\
-8.65 \\
-23.74\end{array}$ \\
\hline $\begin{array}{l}\text { Positive } \\
\text { eigenvalue's } \\
\text { eigenvector }\end{array}$ & & $\begin{array}{l}0.678 \\
0.737 \\
0.000 \\
0.000\end{array}$ & $\begin{array}{l}0.000 \\
0.000 \\
0.846 \\
0.735\end{array}$ & - \\
\hline $\begin{array}{l}\text { Note: The eigen } \\
\text { the type-one sol } \\
\text { component vect } \\
\text { at bus one, whi } \\
\text { magnitude at bus }\end{array}$ & $\begin{array}{l}\text { vector shown is } \\
\text { utions [solutior } \\
\text { correspond to } \\
\text { le the last two } \\
\text { two. }\end{array}$ & $\begin{array}{l}\text { associated wi } \\
\text { is } 1 \text { and } 2 \text { ]. T } \\
\text { the voltage ang } \\
\text { rows correspo }\end{array}$ & $\begin{array}{l}\text { positive } \\
\text { st two ro } \\
\text { radians) } \\
\text { the volt }\end{array}$ & $\begin{array}{l}\text { value for } \\
\text { the four } \\
\text { aagnitude } \\
\text { ngle and }\end{array}$ \\
\hline
\end{tabular}

Table 3-1: Three Bus System Solutions

Solution zero ( $x^{0}$ ) corresponds to the operable solution, while one $\left(x^{1}\right)$ and two $\left(x^{2}\right)$ are type-one low voltage solutions, and three $\left(x^{3}\right)$ is a type-two low voltage solution. The eigenvector associated with the positive eigenvalue for the type-one solutions indicates the area affected by collapse. The presence of non-zero elements in the first two rows of $\mathrm{v}^{1}$ and zero elements in the last two rows of $v^{1}$ indicates that if the load variation was such that voltage collapse occurred by a bifurcation of $x^{0}$ and $x^{1}$, the initial drop in voltage would only occur at bus one (the independence of the bus one and two voltages is due to the presence of the slack bus between them). Thus $\vartheta\left(\mathbf{x}^{1}\right)$ provides a measure of vulnerability to voltage collapse in the area about bus one. Likewise, $\vartheta\left(x^{2}\right)$ provides a measure of vulnerability to voltage collapse in the area of bus two. Since $x^{3}$ is a type-two solution, $\vartheta\left(x^{3}\right)$ is not used. The low value of $\vartheta\left(x^{1}\right)$ indicates that at the given operating point, the bus one area is more vulnerable to collapse than the bus two area. However which area first encounters voltage collapse depends in part upon the relative load participation factors between the two areas. For example, if all subsequent load increase occurs only in area two, $\vartheta\left(\mathrm{x}^{2}\right)$ would decrease more rapidly.

The Stagg and El-Abiad five bus system [22] was chosen as the next test system. Because of system interconnections, the presence or absence of a particular type-one solution is no longer dependent upon the load at a single bus, but rather on system loading as a whole. However, the eigenvector associated with the positive eigenvalue still provides information on the area of collapse for a given type-one solution.

Assume that all real and reactive loads are a linear function of the parameter $k$ ( $k=1$ for basecase). As the value of $k$ is varied, the number of type-one solutions changes. For $\mathbf{k}$ very close to zero the system has four type-one solutions of interest. As $\mathbf{k}$ is increased, the number drops to three at $k=0.03$, to two at $k=$ 0.55 , to one at $k=1.02$, and to zero at the point of voltage collapse $(k=3.36)$ when the operable solution coalesces with the remaining type-one solution. Figure 3-2 plots the variation in the energy measures for each type-one solution with respect to $\mathrm{k}$. Given the smooth variation in the energy measures with respect to changes in system load, system security can be monitored by periodically calculating the type-one solutions and their associated energy measures.

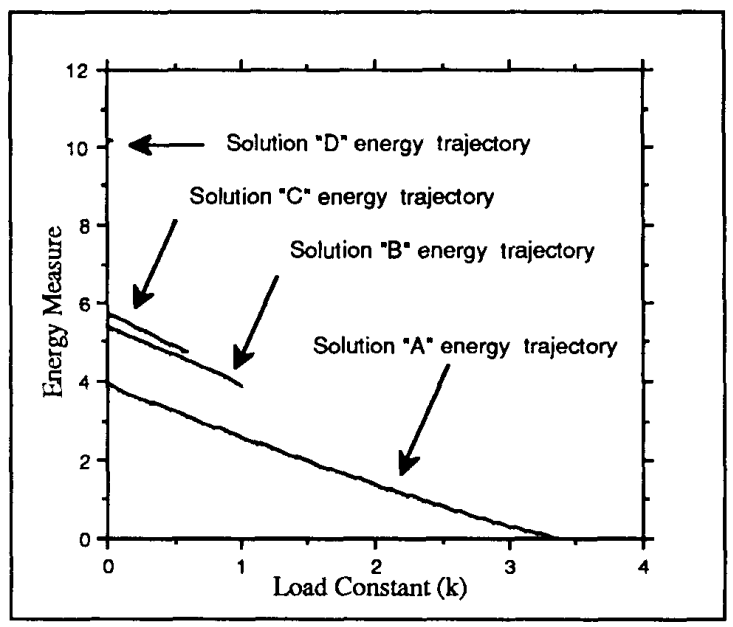

Figure 3-2

As with the three bus system, the eigenvector associated with the positive eigenvalue of the power flow Jacobian provides an interpretation of the multiple type-one solutions. A type-one solution provides a security measure for voltage collapse occurring initially at the buses with the largest eigenvector components. Table 3-2 shows the eigenvectors associated with the positive eigenvalue of the Jacobian for different values of $k$. The eigenvectors are divided into two columns, corresponding to phase and and voltage magnitude. The rows are ordered from bus two through bus five (bus one is the system slack).

\begin{tabular}{|c|c|c|c|c|c|c|c|c|}
\hline & & & one Lo & Volt & Soluti & ons & & \\
\hline k & A & & B & & C & & D & \\
\hline & $\alpha$ & $|v|$ & $\alpha$ & $|v|$ & $\alpha$ & $|v|$ & $\alpha$ & $|v|$ \\
\hline 0.0 & -0.06 & 0.03 & -0.05 & 0.02 & -0.05 & 0.05 & 1.00 & 0.00 \\
\hline & -0.06 & 0.03 & -0.24 & 0.05 & 0.92 & 0.31 & 0.00 & 0.00 \\
\hline & -0.07 & 0.03 & 0.96 & 0.12 & -0.21 & 0.12 & 0.00 & 0.00 \\
\hline & 0.98 & 0.16 & -0.08 & 0.03 & -0.02 & 0.05 & 0.00 & 0.00 \\
\hline 0.5 & -0.07 & 0.05 & -0.06 & 0.04 & -0.07 & 0.06 & * & \\
\hline & -0.06 & 0.05 & -0.20 & 0.10 & 0.93 & 0.32 & & \\
\hline & -0.07 & 0.06 & 0.95 & 0.18 & 0.03 & 0.16 & & \\
\hline & 0.95 & 0.28 & -0.06 & 0.05 & -0.02 & 0.06 & & \\
\hline 1.0 & 0.08 & 0.07 & -0.08 & 0.07 & * & & * & \\
\hline & 0.04 & 0.07 & 0.13 & 0.19 & & & & \\
\hline & 0.06 & 0.09 & 0.94 & 0.21 & & & & \\
\hline & 0.93 & 0.32 & 0.09 & 0.12 & & & & \\
\hline 3.0 & -0.01 & 0.20 & * & & • & & * & \\
\hline & 0.18 & 0.25 & & & & & & \\
\hline & 0.23 & 0.27 & & & & & & \\
\hline & 0.72 & 0.48 & & & & & & \\
\hline & solutio & exists & & & & & & \\
\hline
\end{tabular}

Table 3-2 Eigenvector for Positive Eigenvalue 
The largest component of all the solution $A$ eigenvectors are in rows corresponding to bus 5 quantities. Hence the energy measure for solution A provides an indication of the vulnerability to collapse of an area centered around bus 5 (because of the small size of this system, initially each area contains only a single bus). Likewise solution B energy is an indicator for vulnerability around bus 4 , solution $C$ around bus 3 , and solution $D$ around bus 2 . A precise definition of the buses in a given area is not necessary; only a knowledge of the "area center" (defined as the bus associated with the largest components in the eigenvector). As the load is increased, the areas tend to merge, inferred by the loss of a type-one solution. At some loading level only a single type-one solution and the operable solution remain. To keep the system away from extreme voltage vulnerability, it may be prudent to "track" a number of solutions simultaneously. Then any time an area is moving towards voltage instability appropriate control actions could be identified [14].

Thus the positive eigenvalue's eigenvector provides an interpretation of the energy measure associated with that type-one solution; i.e., the energy measure provides an indicator of vulnerability to voltage collapse in the area of buses with the largest components in the eigenvector. Results in the next section suggest that it is not necessary to calculate the eigenvector; its largest components (i.e. the area center) can be inferred from the power flow initial voltage guess used to calculate the solution.

\section{Efficient Calculation of Appropriate Low Voltage Power Flow Solutions}

As was mentioned earlier, for an $\mathrm{n}$ bus power system, there are believed to be up to $2^{\mathrm{n}-1}$ separate power flow solutions. To use the energy method in an on-line environment, it is imperative that computationally efficient means of determining the appropriate low voltage solutions be employed. In this section such an algorithm is presented.

For the case where just a pair of closely located solutions is desired (e.g. the operable solution and a nearby low voltage solution), the method presented in [12] provides a computationally efficient technique for locating the solutions. This method takes advantage of the convergence characteristic of the rectangular Newton-Raphson method, but requires that the two solutions be closely paired. For the more general situation required here, where a number of low voltage solutions may have to be calculated, an algorithm is presented in [6] that attempts to determine all the low voltage solutions. The algorithm can be summarized as follows. Assume that the operable solution $V^{\mathbf{s}}$ has been calculated. Then for each bus an initial low voltage guess $V_{i}{ }^{u}$ is calculated using a closed form equation [6], with the assumption that the voltages at all other buses are fixed. Then select either $V_{i}{ }^{s}$ or $V_{i}{ }^{u}$ as the initial voltage guesses for the rectangular Newton-Raphson power flow solution. Form all the $2^{n-1}$ possible combinations of initial voltage guess vectors. Attempt to compute power flow solutions for each of the initial guess permutations. The optimal multiplier method [23] is used to prevent power flow divergence or oscillation. While many initial voltage guess vectors had no solutions, the computational effort to attempt power flow solutions for the $2^{n-1}$ guesses proved intractable for all but the smallest systems. A "Simplified Method" was also presented in [6], where only the n-1 combinations of initial voltage guess vectors, using $V_{i}^{\mathbf{u}}$ at each single bus, are calculated.

Extensive numerical tests suggest that the solutions obtained by the Simplified Method correspond to the type-one solutions discussed in the previous section. Additionally the solution found using a low initial guess at bus $i$ has an "area center" at bus $i$. For example, all of the "A" solutions from Table 3-2 and Figure 3-1 were calculated with a low initial guess at bus 5 , the " $B$ " solutions with a low initial guess at bus 4 , the " $C$ " solutions at bus 3 and the " $\mathrm{D}$ " solutions at bus 2 . This suggests the following algorithm:

0 . Obtain the stable operating solution (on-line this is normally available from the state estimator).

1. For each bus $i$ in the system:

a. Calculate $V_{i}^{u}$ and form the power flow initial voltage guess with $V_{j}^{s}$ for $j \neq i$ and $V_{j}^{u}$ for $j=i$.

b. Solve the power flow for the "bus $i$ " solution using the rectangular Newton-Raphson method. If a solution exists calculate the associated energy measure.

Each energy measure then provides an indication of the voltage security in the area of bus $i$. If no solution exists for a low initial guess at bus $i$, then either the bus $i$ area is relatively invulnerable to voltage collapse, or an energy measure exists for a nearby bus.

The solutions with the lowest energy measures indicate the most vulnerable portions of the system. These solutions should be tracked as the system varies with time. If appropriate, system voltage security could be improved by moving controllers in such a way as to increase these energy measures [14]. However solutions with high energy measures would not need to be tracked since they represent secure portions of the system. Therefore voltage security of the entire system can be determined by calculating the relatively few solutions with low energy measures. Thus a drawback of the above algorithm is that in order to determine this small subset of low energy solutions, it is necessary to perform n-1 power flow solutions. This would be computationally prohibitive for a large system. The objective is then to rapidly determine the set of low energy solutions.

An insight into the improvement of the algorithm is suggested by two characteristics of the low energy solutions. First, since by definition the energy measure is an integration from the operable solution to a low voltage solution, the lowest values of energy tend to be associated with the low voltage solutions which are "close" to the operable solution. This is not surprising since if voltage collapse were to occur, it would occur when these two solution ultimately coalesce. Second, the deviation in the bus $i$ solution voltages from the operable solution voltages tends to be localized about bus $\mathrm{i}$. Often the voltages, and hence the power flows in a large portion of a low energy solution do not deviate significantly from the operable solution. Recall that the energy measure is defined as an integration of the power mismatch equations from the operable solution to the low voltage solution. The portion of the energy measure associated with the areas of the system where the two solutions are nearly identical is quite small (i.e. the result of the integration of the power mismatches in that area of the system is quite small). This implies that the bus $i$ energy can be approximated by a partial system solution. The degree of localization is dependent upon the system, but since the low solutions are characterized by higher reactive flows, it is strongly influenced by the location of sources of reactive power.

The proposed solution to the problem of rapidly determining the low energy solutions is to calculate and solve an equivalent system, rather than the full system, for each iteration of the Simplified Method. The equivalent system is created by explicitly retaining bus $i$, along with a set of neighboring buses, and the path set associated with those buses. Adaptive reduction techniques [24] can then be used so that for a large system the computational effort to create each equivalent is substantially less than a single power flow iteration. The accuracy of the energy estimate is dependent upon the number of buses retained. It is 
particularly important that nearby buses with reactive reserve (PV buses) be explicitly retained. The engineering trade-off is between increased accuracy and increased size of the equivalent. For the studies performed here, the algorithm used to determine the set of retained buses explicitly retained all buses up to the second neighbors of bus $i$, and all PV buses up to its fourth neighbors. More sophisticated algorithms could, of course, be used. If a bus i low voltage solution exists for the equivalent system, an equivalent energy measure is calculated. Full low voltage solutions are calculated only for those buses whose equivalent energy is sufficiently low. If the equivalent system contains no bus $i$ solution, then it is assumed that the full system also does not possess a bus i solution. Desirable properties of the equivalent are first that it have a bus i solution if and only if the full network has a bus i solution, second that if a solution exists, the equivalent's energy accurately estimate the energy of the full solution, and third that the size of the equivalent network be sufficiently small. The Ward Admittance equivalence method from [25] was chosen. As will be seen in the following examples, this method provides good correlation between the equivalent solution energy and the full solution energy. Additionally, errors tend to be in the conservative direction of underestimating the value of the true solution energy. The following "Improved Method" is proposed:

0 . Obtain the stable operating solution.

1. The Ybus matrix is re-ordered and factored.

2 . For each bus $i$ in the system (Screening stage)

a. Determine the set of retained buses (including bus i) and their path set; then using adaptive reduction setup an equivalent system.

b. Calculate $V_{i}{ }^{u}$ and form the initial voltage guess for all buses in the equivalent with $V_{j}{ }^{s}$ for $j \neq i$ and $V_{j}{ }^{u}$ for $j=i$.

c. Solve the equivalent system using the rectangular Newton-Raphson method.

d. If a solution exists calculate its energy measure. If energy measure is sufficiently small, store solution.

3. For each bus with a sufficiently small energy measure for the equivalent system (Solution stage)

a. Form the power flow initial voltage guess using $V_{j}^{s}$ for $j$ not in the equivalent and $V_{j}$ (eqv) for $j$ in equivalent.

b. Solve the full system using the rectangular NewtonRaphson method.

c. If a solution exists calculate its energy measure.

In the first step, the matrix was re-ordered in order to minimize the length of the factorization paths used in the adaptive reduction. This reordering was done because numerical testing between Tinney Scheme 2 and the Minimum Degree Minimum Length (MDML) algorithm from [26] indicated that smaller equivalent networks tended to result when the MDML method was used. The number of buses explicitly retained in each equivalent is independent of the original system size; the number of buses in the path set depends on the structure of the factorization path graph.

The algorithm was tested on the IEEE 118 bus case. For the studies performed here, loads were assumed to be a linear function of a parameter $\mathrm{k}$ ( $\mathrm{k}=1$ for basecase). Reasonable generator participation factors were used. The upper curve in Figure 4-1 shows the variation in number of low voltage solutions (calculated using the Simplified Method) with respect to $\mathrm{k}$. As the system load increases, the number of solutions tends to decrease. The lower curve in the figure shows the number of solutions with energy values below 3.0. For low load levels, when the system is quite secure, there are only a few solutions with energy values less than 3.0 (the lowest energy value for $k=1$ is 2.75 ). As the system loading increases, the number of low energy solutions also tends to increase, indicating that the system is becoming more vulnerable to voltage instability in a number of areas. However, the number of low energy solutions remains quite moderate. Immediately before voltage collapse there is only a single low voltage solution. Convergence properties of the Newton-Raphson method unfortunately make it possible that a low initial guess at bus $j$ converges to the bus $k$ solution (with $j \neq k$ ). However, in the thousands of powerflows performed to produce Figure 4-1, this occurred only a few times.

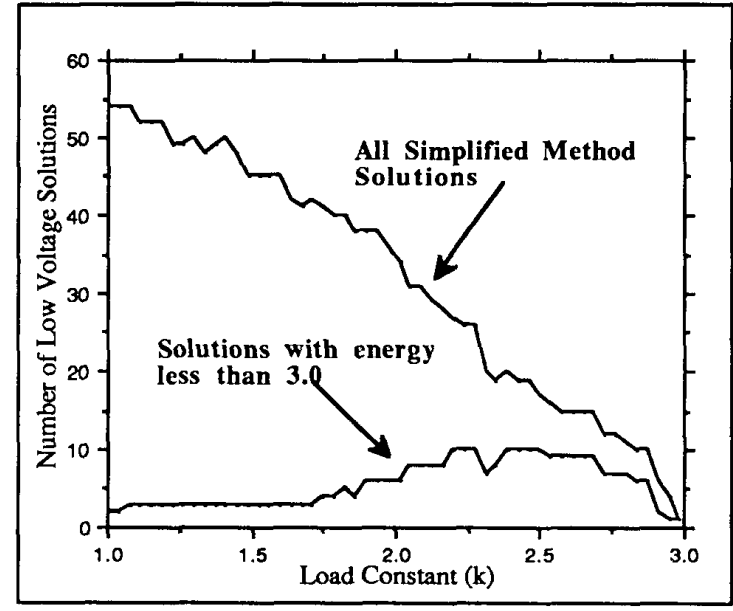

Figure 4-1

\begin{tabular}{|lcc|}
\hline Order/Factor Ybus & Simplified Method & Improved Method \\
Construct 117 Equivalents & - & 0.88 \\
for Screening & & 52.79 \\
$\begin{array}{l}\text { Solution of } 117 \text { equiv. systems } \\
\text { and screening energy }\end{array}$ & - & 189.27 \\
$\begin{array}{l}\text { Full Solutions and } \\
\text { energy calculations }\end{array}$ & 570.84 & 51.74 \\
Total & 570.84 & 294.68 \\
\hline
\end{tabular}

Table 4-1 - Comparison of Computational Efficiency

The computational efficiency of the Improved Method is demonstrated by comparing it to the Simplified Method in Table 41. Simulations shown are for $k=1$, and times given are in seconds. For reference, the time necessary to calculate the operable power flow solution from a flat start was 3.18 seconds on the machine used.

To determine the set of low energy solutions at each time step using the Simplified method required 117 power flow solutions of the full IEEE 118 bus system (the system has 186 lines). For the Improved Method the ordering and factoring of the Ybus only had to be performed once. Most of the time was spend solving the equivalent systems. The average size of the equivalent systems was 31 buses with 60 lines. Full solutions were then only performed for the ten buses with lowest screened energy values.

The accuracy of the Improved Method is demonstrated by comparing the equivalent system energies to the energy obtained by the Simplified method. At $k=1,54$ low voltage solutions were found using the Simplified method, while 45 were found for the equivalent systems. Of these 45 , only one did not have a corresponding full system solution (a "false alarm"). All of the 10 solutions missed by screening had energies greater than 5.0 and therefore none were considered low energy. Table 4-2 compares the energy of the equivalent systems to the full solution energies 
for the ten lowest values. Note that the ranking of the equivalent energies correlates quite closely to the ranking of the full solution energies, and that the error is in the conservative direction of underestimating the true value.

\begin{tabular}{|ccc|}
\hline Bus \# & Equivalent System Energy & Full System Energy \\
10 & 2.21 & 3.61 \\
21 & 2.40 & 3.03 \\
44 & 2.67 & 2.75 \\
43 & 2.71 & 2.81 \\
20 & 2.81 & 3.75 \\
107 & 3.11 & solution \\
22 & 3.39 & 3.52 \\
9 & 3.42 & 3.61 \\
76 & 3.83 & 4.01 \\
53 & 3.85 & 3.85 \\
\hline
\end{tabular}

The tests were repeated at a higher value of load $(k=2.5)$. Thirteen of the seventeen solutions found using the Simplified method were identified during screening. These thirteen included all of the nine solutions with energy less than 3.0.

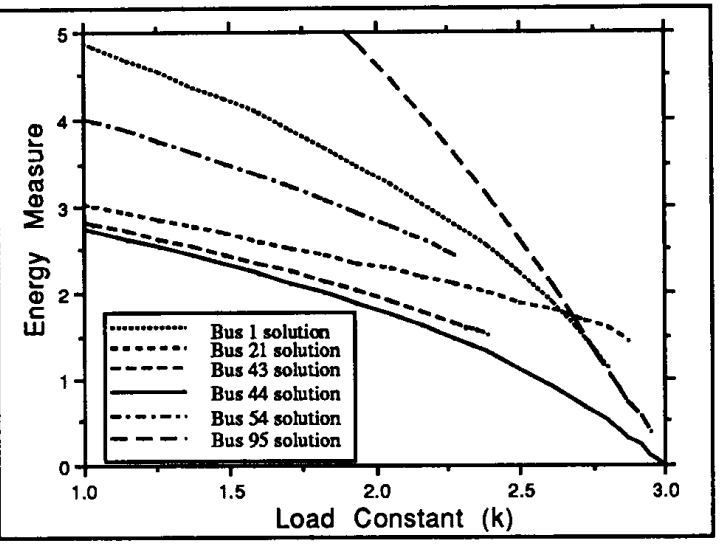

Figure 4-2

Figure 4-2 plots the variation in the energy with respect to $k$ for the six solutions having lowest energy. The various energy measures provide an indication of the most vulnerable areas of the system. For low load levels the high energy levels indicate that the system is relatively secure, with the weakest areas in the vicinity of buses 44,43 , and 21 . Again, each energy quantity provides a measure of voltage vulnerability in a particular area, as can be verified by calculating the eigenvector for the positive eigenvalue of the Jacobian. For example, in the bus $\mathbf{4 4}$ solution, the largest eigenvector components were associated with bus 44 , with other significant component values (5\% of bus 44 values) found only at its first and second neighbor buses.

As the load is increased, the energy measures tend to decrease. The rate of decrease is dependent upon the rate of change of the system parameters. For example while the area in the vicinity of bus 95 is quite secure initially, the high subsequent load increase (due to its large basecase load) causes a rapid drop in its voltage security and hence its energy measure. This underscores the necessity of monitoring more than just the lowest energy solution. For any given system state, the energy measures provide relative ranking of the voltage security of the areas of the system. When combined with their rate of change, the energy values provide the system operator with a very good indication of how close the system is to voltage collapse and where collapse would initially occur. The rate of change information could either be determined by monitoring the time variation of the energy values; or when accurate future load/generation participation estimates are available, computed analytically using techniques from [14].

Lastly the Improved Method was tested on a 415 bus/609 line system. The average size of the equivalents used in the screening was 37 buses and 94 lines. Thus, comparing to the 118 bus case, a $300 \%$ increase in system size has resulted in only about a $50 \%$ increase in equivalent system size. The average time to build and solve each equivalent system was about $20 \%$ of a power flow solution of the full system. Full solutions were performed for the ten buses with the lowest screening energy values. Comparison with results of the Simplified Method indicated that screening correctly located the nine buses with the lowest energies, with energy errors again in the conservative direction.

\section{v. Conclusions}

This paper has introduced an algorithm for rapidly determining the low voltage power flow solutions with the smallest associated energy measures. The algorithm was then illustrated in conjunction with energy methods to assess power system security with respect to voltage instability. Energy methods offer the advantages of smooth variation with respect to system changes, and properties of the low voltage solutions identify particular areas of the system most vulnerable to voltage collapse. Previous research had identified efficient calculation of low voltage solutions as the main impediment in the application of energy methods to the voltage collapse problem. The Improved Method presented here offers a viable solution to this difficulty. In conclusion, energy methods, coupled with the Improved Method for low voltage power flow solutions, provide a good method of assessing power system security with respect to voltage problems.

\section{Acknowledgements}

The support of this work by the Electric Power Research Institute Project RP 2675-4 and National Science Foundation grant ECS-8857019 is gratefully acknowledged.

\section{Appendix}

The energy function used in this paper for the determination of voltage security is defined as:

$$
\vartheta\left(x^{s}, x^{u}\right)=\int_{\left(\alpha^{s}, v^{s}\right)}^{\left(\alpha^{u}, v^{u}\right)}\left[f^{T}(\xi, v), g^{T}(\xi, v)\right]^{T}[d \xi, d v]^{T}
$$

where $x^{s}:=\left(\alpha^{s}, V^{s}\right)$ is the Stable Equilibrium Point (SEP), $x^{u}:=$ $\left(\alpha^{\mathrm{u}}, V^{\mathrm{u}}\right)$ an Unstable Equilibrium Point (UEP), $\mathrm{V}$ and $\alpha$ are the system voltage magnitudes and angles, and

$$
\begin{aligned}
& f_{i}(\alpha, V)=P_{i}-\sum_{j=1}^{n} B_{i j}\left|v_{i}\right|\left|v_{j}\right| \sin \left(\alpha_{i}-\alpha_{j}\right) \\
& -\sum_{j=1}^{n} G_{i j}\left|v_{i}^{s}\right|\left|v_{j}^{s}\right| \cos \left(\alpha_{i}^{s}-\alpha_{j}^{s}\right) \\
& g_{i}(\alpha, V)=\left(V_{i}\right)^{-1}\left(Q_{i}+\sum_{j=1}^{n} B_{i j}\left|v_{i}\right|\left|v_{j}\right| \cos \left(\alpha_{i}-\alpha_{j}\right)\right) \\
& -\left(V_{i}^{s}\right)^{-1} \sum_{j=1}^{n} G_{i j}\left|v_{i}^{s}\right|\left|v_{j}^{s}\right| \sin \left(\alpha_{i}^{s}-\alpha_{j}^{s}\right)
\end{aligned}
$$


The constant terms on the second lines of (A2) and (A3) are included so that $f$ and $g$ are identically zero at the SEP solution even for networks with non-zero transfer conductance terms. Evaluating the integral (A1) from $x^{s}$ to $x^{4}$, the energy function is given as:

$$
\begin{aligned}
& \vartheta\left(x^{s}, x^{u}\right)=-\frac{1}{2} \sum_{i=1}^{n} \sum_{j=1}^{n} B_{i j}\left|v_{i}^{u}\right|\left|v_{j}^{u}\right| \cos \left(\alpha_{i}^{u}-\alpha_{j}^{u}\right) \\
& +\frac{1}{2} \sum_{i=1}^{n} \sum_{j=1}^{n} B_{i j}\left|v_{i}^{s}\right|\left|v_{j}^{s}\right| \cos \left(\alpha_{i}^{s}-\alpha_{j}^{s}\right) \\
& +\sum_{i=1}^{n} v_{i}^{s} \frac{Q_{j}(x)}{x} d x-P^{T}\left(\alpha^{u}-\alpha^{s}\right) \\
& -\sum_{i=1}^{n}\left(\sum_{j=1}^{n} G_{i j}^{u}\left|v_{i}^{s}\right|\left|v_{j}^{s}\right| \cos \left(\alpha_{i}^{s}-\alpha_{j}^{s}\right)\left(\alpha_{i}^{u}-\alpha_{i}^{s}\right)\right) \\
& +\sum_{i=1}^{n}\left(\left(v_{i}^{s}\right)^{-1} \sum_{j=1}^{n} G_{i j}\left|v_{i}^{s}\right|\left|v_{i}^{s}\right| \sin \left(\alpha_{i}^{s}-\alpha_{j}^{s}\right)\left(v_{i}^{u}-v_{i}^{s}\right)\right.
\end{aligned}
$$

Since in the voltage security context one is only concemed with the energy differences between equilibrium points, (A1) and (A4) are only potential energy components of a total system energy function. In order for (A1) to formally define a Lyapunov function, the kinetic energy term would have to be included and a number of restrictions placed on allowable system dynamic models. These would include 1) not allowing voltage dependence in the real power load, 2) restricting the method to networks with no transfer conductance terms. In the realistic systems considered here, these restrictions are relaxed, and hence we use the term "energy function" rather than Lyapunov function.

\section{References}

[1] A. Kurita and T. Sakurai, "The Power System Failure on July 23, 1987 in Tokyo", Proc. 27th IEEE Conf. Decision and Control, Austin, TX, Dec 1988.

[2] C. Barbier and J-P Barret, "Analysis of Phenomena of Voltage Collapse on a Transmission System," Revue Generale de l'electricite, Vol. 89, Oct. 1980, pp. 672-680.

[3] G.A. Cucchi, "Voltage Stability and Security, the Operator's View: 'Seeing More Now but Enjoying it Less'," Engineering Foundation Conf. on Bulk Power System Voltage Phenomena. Voltage Stability and Security," Potosi, MO, Sep. 1988.

[4] R.K. Gupta et al.,"Steady State Voltage Instability Operations Perspective," IEEE PES Winter Meeting, WM 037-2, Atlanta, GA, Feb. 1990.

[5] A. Klos and A. Kerner, "The non-uniqueness of Load-flow Solution," Proc. PSCC V 3.1/8, Cambridge, 1975

[6] Y. Tamura, K. Iba, and S. Iwamoto, "A Method for Finding Multiple Load-flow Solutions for General Power Systems," Proc IEEE PES Winter Meeting, A80 043-0, New York, Feb. 1980.

[7] Y. Tamura, H. Mori, and S. Iwamoto, "Relationship between Voltage Instability and Multiple Load Flow Solutions in Electric Power Systems," IEEE Trans. Power App. and Sys. Vol. PAS-102, pp. 1115-1123, May 1983.

[8] A. Araposthatis, S. Sastry, and P. Varaiya, "Analysis of the Powerflow Equation," Int. J. of Electrical Power Energy Systems, Vol. 3, pp. 115-126, July 1981.

[9] H.G. Kwatny, A. K. Pasrija, and L.Y. Bahar, "Static Bifurcations in Electric Power Networks: Loss of Steady-State Stability and Voltage Collapse," IEEE Trans. Circuits and Systems, Vol. CAS-33, pp. 981-991, Oct. 1986.
10] Y. Tamura et al., "Current Issues in the Analysis of Voltage Instability Phenomena," Engineering Foundation Conference on Bulk Power System Voltage Phenomena: Voltage Stability and Security," Potosi, MO, Sep. 1988.

[11] A. Yokoyama and Y. Sekine, "A Static Voltage Stability Index Based on Multiple Load Flow Solutions," Engineering Foundation Conf. on Bulk Power System Voltage Phenomena: Voltage Stability and Security," Potosi, MO, Sep. 1988.

[12] K. Iba et al., "A Method for Finding a Pair of Multiple Load Flow Solutions in Bulk Power Systems," IEEE Trans. on Power Sys., Vol. PWRS-5, pp. 582-591, May 1990.

[13] C. L. DeMarco and T. J. Overbye, "An Energy Based Security Measure for Assessing Vulnerability to Voltage Collapse," IEEE Trans. on Power Sys., Vol. PWRS-5, pp. 419 427. May 1990.

[14] T. J. Overbye and C. L. DeMarco, "Voltage Security Enhancement using Energy Based Sensitivities", IEEE PES Summer Meeting, SM 478-8, Minneapolis, MN, July 1990.

[15] J. Baillieul and C. I. Bymes, "Geometric Critical Point Analysis of Lossless Power System Models," IEEE Trans. Circuits and Systems, Vol. CAS-29, pp. 724-737, Nov. 1982.

[16] Y. Tamura et al., "Monitoring and Control Strategies of Voltage Stability based on Voltage Instability Index," Engineering Foundation Conf. on Bulk Power System Voltage Phenomena: Voltage Stability and Security," Potosi, MO, Sept. 1988.

[17] Y. Tamura, K. Sakamoto, and Y. Tayama, "Voltage Instability Proximity Index (VIPI) Based on Multiple Load Flow Solutions in Ill-Conditioned Power Systems," Proc. 27th IEEE Conf. Decision and Control, Austin, TX, Dec. 1988.

[18] I. Dobson and H. Chiang, "Towards a Theory of Voltage Collapse in Electric Power Systems," Systems \& Control Letters, pp. 253-262, 1989.

[19] P.W. Sauer and M.A. Pai, "Power System Steady State Stability and the Load-Flow Jacobian," IEEE Trans. on Power Sys. Vol. PWRS-5, pp. 1374-1383, Nov, 1990

[20] C.L. DeMarco, "Probabilistic Dynamic Security Measures for Analyzing Voltage Stability," Engineering Foundation Conference on Bulk Power System Voltage Phenomena: Voltage Stability and Security," Potosi, MO, Sep. 1988.

[21] I. Dobson et al., "A Model of Voltage Collapse in Electric Power Systems," Proc. 27th IEEE Conf. Decision and Control, Austin, TX, Dec. 1988.

[22] G.W. Stagg and A.H. El-Abiad, Computer Methods in Power Systems Analysis, McGraw-Hill, New York, NY, 1968.

[23] S. Iwamoto and Y. Tamura, "A Load Flow Calculation for Ill-conditioned Power Systems," IEEE Trans. Power App. and Sys., Vol. PAS-100, pp. 1736-1743, April 1981.

24] W.F. Tinney and J.M. Bright, "Adaptive Reductions for Power Flow Equivalents," IEEE Trans. on Power Sys., Vol. PWRS-2, pp. 351-360, May 1987.

[25] S. Deckmann et al., "Numerical testing of power system load flow equivalents," IEEE Trans. Power App. and Sys., Vol. PAS-99, pp. 2292-2300, Nov/Dec 1980.

[26] R. Betancourt, "An efficient heuristic ordering algorithm for partial matrix refactorization," IEEE Trans. on Power Sys., Vol. PWRS-3, pp. 1181-1187, Aug. 1988.

Thomas J. Overbye (S '87) was born in Milwaukee, WI in 1960 He received his Bachelor of Science degree from the University of Wisconsin-Madison in 1983. He was employed with Madison Gas and Electric Company from 1983 through 1986. He is currently pursuing a $\mathrm{PhD}$ in Electrical Engineering at the University of Wisconsin-Madison.

Christopher L. DeMarce (S '80, M '85) was born in Derby, Connecticut in 1958. He received his Bachelor of Science degree from M.I.T. in June of 1980, and his Ph.D. degree in Electrical Engineering and Computer Sciences from the University of California, Berkeley in May 1985. In January 1985, he joined the faculty of the Department of Electrical and Computer Engineering at the University of Wisconsin-Madison, where he is currently an Associate Professor. 